- Dorothy House Hospice Care physio continues to provide specialist treatment and advice for those who need it.

- Consideration of seven-day week support (funding dependent).

\section{P-158 COLLABORATION BETWEEN STARLIGHT REHABILITATION SERVICE AND LOCAL EVERYONE ACTIVE GYM}

Jo Marovitch. Peace Hospice Care, Watford, UK

\subsection{6/bmjspcare-2019-HUKNC.180}

Background The Hospice Rehabilitation Service aims to maintain independence, build confidence, improve strength, reduce fatigue, reduce side effects of treatments, depression and anxiety. The benefits of exercise for people living with long-term conditions, both physically, emotionally and psychologically, are widely recognised.

Aims A local gym agreed to provide a Personal Trainer free of charge to run a group under the guidance of hospice staff. The project aims to:

- Enable and empower active and independent patients to access a local community gym for independent exercise;

- Improve patients' health and wellbeing.

Participating patients would then be offered gym membership at a reduced rate on completion of the programme.

Method Patients set their goals, and hospice staff provided referrals, relevant information and training in rehabilitation principles and fatigue to the Personal Trainer. The patients were taken as a group into the gym environment to access the equipment safely at their own level, building their confidence in small steps. Seven patients were referred, and the Personal Trainer developed programmes suited to individual needs.

Results Four completed (57\%) and reported increased strength, mobility and fitness, weight loss and improved confidence. Two of the group have now taken up gym membership and one has achieved his goal to get back onto the golf course.

Conclusion The programme had a positive impact on our patients' health and wellbeing and the hospice has successfully enabled patients to access community resources. The second cohort of patients have been referred and progress is being monitored.

'Exercise not only changes your body, it changes your mind, your attitude and your mood.'

\section{P-159 IMPROVING ACCESS AND OPPORTUNITIES TO EXERCISE FOR PALLIATIVE PATIENTS}

Colette Parfitt. Marie Curie Hospice, Liverpool, UK

\subsection{6/bmjspcare-2019-HUKNC.181}

Background Research suggests that participating in some form of physical activity improves quality of life and leads to better outcomes for palliative patients. However, evidence suggests that there are variable levels of participation and engagement in physical activity for many reasons. One of the common reasons provided is the reluctance to access mainstream gyms and services due to a perceived lack of support and specialist knowledge of their diagnosis and symptoms. Due to our small team, there are often many challenges to providing our patients with these services.

Aims Our aim was to improve the opportunities for hospice patients to participate in supported gym-based exercise groups. Methods We contacted several universities to establish interest in exercise student placements. We developed links with a local university, arranging clinical placements for six exercise students over an eight week period.

Following an initial physiotherapy assessment, and prescription of appropriate exercises, two students worked together delivering gym sessions. Students were encouraged to progress exercises with physiotherapy support and report any issues back to the therapy team.

Following the eight weeks, feedback was obtained from both students and patients.

Results Results have been positive. The students have enjoyed the experience a clinical placement has offered, and two have since enrolled onto a physiotherapy degree course. Uptake onto the classes and gym sessions was good. As well as reporting enjoyment of the sessions, patients reported improvements in quality of life.

Conclusion Our work has demonstrated that offering exercise services within a hospice is important to palliative patients. Developing relationships with other institutions and exploring the use of different academic students (to complement our physiotherapy student placements) is both resourceful and valuable. Work is ongoing. We hope that by maintaining these links we will provide hospice patients with greater opportunity to access beneficial services for the future.

\section{P-160 A PILOT STUDY TO MEASURE OUTCOMES OF USING A REHABILITATIVE PALLIATIVE CARE PHYSIOTHERAPY SERVICE}

Dawn Tidmarsh, Sarah Bradfield. Garden House Hospice Care, Letchworth, UK

\subsection{6/bmispcare-2019-HUKNC.182}

Background Having in the past two and a half years changed our day service delivery from a traditional day hospice to an out-patients supportive rehabilitative care service we wanted to formally measure the outcomes for patients using our physiotherapy and gym programme. Anecdotally patients reported 'feeling better and fitter' but this wasn't fully captured by reviewing their gym programme alone. We hypothesised that in a group of patients with an overall declining picture of health we needed to look at not only their physical condition, but also the potential mental health benefits of exercise. We believed that even if patients were physically declining, we could use the mental health questionnaire results to encourage and motivate patients to continue with their programmes.

Aims Our aim was to pilot a system whereby patients completed an IPOS, identified a goal they wanted to be able to achieve (e.g. 'to be able to walk to town') and completed an anxiety and depression questionnaire.

Methods From late 2018, existing and new patients to the gym have completed an IPOS, identified a specific and measurable goal and have been asked to complete the PHQ-9 (Depression) and GAD-7 (Anxiety) questionnaires. Patients are then reviewed approximately every 12 weeks. Results of the review and questionnaires are collated and used to identify not only further physiotherapy intervention but whether the 